\title{
LABORATÓRIO DE EXERGAMES: UM ESPAÇO COMPLEMENTAR PARA AS AULAS DE EDUCAÇÃO FÍSICA
}

\author{
EXERGAMES LAB: A COMPLEMENTARY SPACE FOR PHYSICAL \\ EDUCATION CLASSES
}
LABORATORIO DE EXERGAMES: UN ESPACIO COMPLEMENTARIO PARA LAS CLASES DE EDUCACIÓN FÍSICA

Mateus David Finco*, Eliseo Berni Reategui ${ }^{*}$, Milton Antonio Zaro*

\section{Palavras-chave Jogos de vídeo. Comportamento cooperativo. Relações interpessoais.}

\begin{abstract}
Resumo: Este artigo apresenta um estudo sobre o uso de exergames em práticas estruturadas em aulas de Educação Física envolvendo estudantes que geralmente apresentam sinais de insatisfação com atividades esportivas propostas nas aulas regulares da disciplina. Enquanto os videogames foram historicamente associados a problemas como obesidade e introversão social, os exergames trouxeram uma nova perspectiva para esses artefatos culturais. Vinte e quatro alunos entre oito e 14 anos de idade participaram do estudo, realizado em um laboratório criado em uma escola no sul do Brasil. Os alunos foram observados e seus professores de Educação Física entrevistados ao final do projeto. Como resultado, foi possível observar que os estudantes que normalmente não demonstravam interesse pelas aulas de Educação Física passaram a apresentar uma atitude positiva em relação às práticas com os exergames, evidenciando também sua disposição em colaborar com os colegas.
\end{abstract}

Abstract: This paper presents a study about the use of exergames in structured Physical Education (PE) classes with students that usually show signs of dissatisfaction with sports activities proposed at regular PE classes. While videogames have been historically associated with issues related to obesity and social introversion, exergames brought a new perspective on those cultural artifacts. Twenty-four 8-14-year-old students participated in the study conducted in a laboratory set up in a school in Southern Brazil. They were observed and their PE teachers were also interviewed at the end of project. Results showed that students that usually did not show interest in Physical Education classes started to demonstrate a positive attitude regarding exergame practices, also expressing willingness to cooperate with their peers.

Resumen: Este artículo presenta un estudio sobre el uso de exergames en prácticas estructuradas en clases de Educación Física con estudiantes que generalmente presentan señales de insatisfacción frente a las actividades deportivas propuestas en las clases regulares de la asignatura. Mientras los videojuegos han sido históricamente asociados a problemas como obesidad e introversión social, los exergames han traído una nueva perspectiva para esos artefactos culturales. Veinticuatro alumnos entre ocho y catorce años de edad han participado en este estudio, desarrollado en un laboratorio organizado en una escuela en el sur de Brasil. Los estudiantes fueron observados y sus profesores de Educación Física entrevistados al final del proyecto. Como resultado, fue posible observar que los estudiantes que normalmente no mostraban interés por las clases de Educación Física, pasaron a presentar una actitud positiva en relación a las prácticas con los exergames, evidenciando también su disposición de colaborar con sus compañeros.
*Universidade Federal do Rio Grande do Sul. Porto Alegre, RS, Brasil. E-mail: mateusfinco@gmail.com

Recebido em: 17-12-2014

Aprovado em: 05-06-2015 


\section{INTRODUÇÃO}

Nos dias de hoje, crianças e adolescentes estão submersos numa sociedade em que a tecnologia permeia praticamente todos os segmentos. Neste contexto, os jogos digitais se constituem em um dos artefatos tecnológicos mais empregados. Videogames ativos, ou exergames, foram introduzidos como uma forma de permitir que o corpo pudesse controlar tais jogos. Como resultado, esses jogos passaram a ser vistos como uma ferramenta que poderia auxiliar as pessoas a adotar um estilo de vida menos sedentário (TROUT; CRISTIE, 2007), com efeitos positivos sobre o comportamento relacionado à saúde (BARANOWSKI, 2008). Mas, embora haja controvérsias sobre a capacidade desses jogos de melhorar a aptidão das crianças de acordo com as recomendações de saúde pública, tem-se argumentado que os exergames podem contribuir para a prática regular de atividade física moderada (PENG; LIN; CROUSE, 2011). Promovendo a interação entre jogadores, os exergames também podem contribuir para a redução do sentimento de isolamento social e a solidão (MUELLER; AGAMANOLIS; PICARD, 2003).

A Educação Física é a disciplina no currículo escolar voltada à prática de atividade física, entre outros objetivos. Pesquisadores têm enfatizado a importância de crianças em idade escolar realizarem diariamente exercícios físicos com intensidade moderada a vigorosa, envolvendo diferentes atividades (STRONG et al., 2005). Com base em tal evidência, 0 Departamento Americano de Saúde e Serviços Humanos (US DEPARTMENT OF HEALTH AND HUMAN SERVICES, 2009) afirma que os programas de atividade física escolar devem ajudar a alcançar tais metas, salientando que as práticas de Educação Física devem ser agradáveis e envolver as crianças em um tipo de interação amigável.

Neste contexto, surge a necessidade de propor novas alternativas para a prática de exercício físico, buscando atender recomendações de que as práticas de Educação Física sejam aprazíveis para todos os alunos. A apreciação pela prática não apenas pode aumentar o nível da atividade física (DISHMAN et al., 2005), como também pode influenciar a adesão às atividades a longo prazo (WILLIAMS et al., 2006). Além disso, embora a atitude do estudante com relação à Educação Física seja geralmente positiva, as diferenças individuais podem levar os alunos a não se motivarem a participar das aulas (STELZER et al., 2004). Dados apontam que, em alguns casos, até $20 \%$ dos alunos não gostam das aulas de Educação Física (CARLSON, 1995). Determinadas experiências em Educação Física baseadas em práticas esportivas já apresentaram um impacto negativo sobre a percepção dos indivíduos quanto à prática de atividade física (ENNIS, 1996). Nessas experiências, os alunos de ambos os sexos relataram um intenso desagrado com relação às práticas desenvolvidas. Portanto, é importante que o currículo da Educação Física Escolar possa incorporar diferentes tipos de atividade que sejam percebidas como aprazíveis para todos os alunos. Esta tem sido a proposta do projeto apresentado neste artigo, que se concentrou na criação de um laboratório de exergames como subsídio às aulas regulares de Educação Física.

Além dos benefícios orientados para a saúde, o envolvimento dos estudantes em atividades físicas também pode oferecer oportunidades para a interação social, contribuindo para o desenvolvimento de habilidades de trabalho em equipe e a capacidade de cooperar. Evidências científicas têm demonstrado que programas de Educação Física bem estruturados podem contribuir para a melhoria das competências sociais das crianças e adolescentes, 
podendo envolvê-los em diversas situações sociais, tais como a cooperação, a assistência, a troca de experiências e a resolução de problemas (GREGORIADIS; GRAMMATIKOPOULOS; ZACHOPOULOU, 2013).

Neste artigo, buscou-se compreender de que maneira o uso de exergames pode contribuir com a prática de atividade física e com o desenvolvimento de habilidades sociais. Centrando-se nesses aspectos, buscou-se entender como a prática com esses jogos em pequenos grupos pode envolver crianças e adolescentes desmotivados em participar de aulas regulares de Educação Física.

\section{EXERGAMES NAS AULAS DE EDUCAÇÃO FÍSICA}

A maior parte da literatura científica relacionada aos exergames e educação se concentra no potencial do jogo para melhorar a saúde física dos alunos, envolvê-los em atividades sociais e melhorar seu desempenho acadêmico (STAIANO; CALVERT, 2011). Resultados de pesquisas recentes também têm mostrado que tais jogos podem contribuir para o treinamento de práticas esportivas e outras atividades envolvendo movimento (FERY; PONSERRE, 2011), ou para o desenvolvimento de habilidades motoras (HAYES; SILBERMAN, 2007). Por outro lado, tem-se argumentado que os exergames não podem ser um substituto para "esportes reais" e que podem não favorecer um nível motivacional alto a longo prazo para a prática sustentável de atividades físicas (WIEMEYER, 2010). Tais posições díspares em relação aos exergames mostram a necessidade de realização de novas investigações sobre a forma como esses jogos podem ser usados em ambientes formais de educação.

Vários estudos estão sendo realizados neste sentido, alguns deles com resultados conclusivos sobre os benefícios dos exergames. Por exemplo, Shayne et al. (2012) compararam os efeitos da Educação Física tradicional e dos exergames na prática de atividade física entre quatro crianças ativas. Enquanto na aula de Educação Física o professor conduziu as atividades de acordo com os planos de aula regulares, no laboratório de exergames os estudantes jogaram com uma série de jogos de acordo com certas regras estabelecidas para a prática. Os resultados mostraram que os exergames produziram maior percentual na prática de atividade física e representaram uma oportunidade diferente para envolver os alunos em atividades sociais. Em outro estudo, Sun (2013) observou que o uso prolongado de exergames levou um grupo de crianças do ensino básico a um maior interesse por atividades físicas, tanto para meninos quanto para meninas. Tais resultados levaram o autor a recomendar as práticas com exergames como um possível método alternativo nas práticas em Educação Física.

Alguns estudos tentaram mostrar como exergames também podem contribuir para o desenvolvimento de habilidades afetivas nas práticas em Educação Física. Lieberman (2006) descreveu diferentes fatores que podem promover a aprendizagem através de jogos, incluindo características que os tornam um meio adequado para a interação social: o reconhecimento social para habilidades de jogo, interação social para melhorar as comunidades de aprendizagem e jogos como ambientes de aprendizagem. Um estudo com um jogo de simulação de dança também mostrou que as crianças que participaram em atividades com vários jogadores apreciaram a interação com seus pares e tiveram uma taxa de abandono inferior, jogando cerca de duas vezes mais que os estudantes que jogaram o jogo por conta própria (CHIN et al., 2008). 
O estudo aqui descrito apresenta uma experiência com um laboratório de exergames estruturado como um espaço complementar para as aulas de Educação Física. O trabalho apresenta o laboratório como um espaço educativo voltado principalmente a crianças e préadolescentes desmotivados a participar de aulas regulares de Educação Física. $O$ objetivo do laboratório foi de incentivar a prática de exercícios físicos e de permitir que as crianças também trabalhassem suas habilidades sociais.

\section{METODOLOGIA}

Esta seção apresenta o relato da experiência com a criação do laboratório de exergames. O laboratório foi criado em uma escola particular de ensino fundamental e médio no sul do Brasil, equipado com um Wii/Wii Fit e um console Xbox/Kinect, mas apenas o Xbox/ Kinect foi usado para o objetivo desta pesquisa. Dois títulos comerciais foram selecionados para o projeto (Kinect Sports e Kinect Adventures) em razão da grande variedade de atividades que esses dois jogos apresentam, incluindo jogos de esportes (tênis de mesa, atletismo, vôlei de praia, boliche e boxe), assim como outros jogos que exigem a realização de diferentes movimentos corporais, como saltar, chutar, dançar e pisar.

Vinte e quatro alunos de ambos os sexos participaram do experimento, sendo 12 do sexo masculino e 12 do sexo feminino. As reuniões tiveram duração de uma hora, possibilitando a participação de no máximo quatro alunos por grupo, de acordo com as faixas etárias: 8-9, 10-11, 12-14 anos de idade. Os participantes do projeto foram selecionados por uma comissão composta pelo coordenador pedagógico da área de Educação Física, dois membros da equipe de pesquisa da universidade envolvida no projeto e quatro professores de Educação Física.

A principal faceta usada para selecionar os alunos foi sua falta de motivação para participar das aulas, um problema com a natureza multidimensional que pode envolver fatores como sedentarismo, obesidade, dificuldades motoras, introversão ou simplesmente um descontentamento com as atividades propostas nas aulas regulares. Utilizando as próprias palavras de um dos professores de Educação Física envolvidos no projeto, os alunos que foram convidados a participar foram os

[...] que talvez não gostem muito, os que ficam um pouco relutantes em participar das aulas regulares de Educação Física. Nós enviamos os estudantes que pensamos que poderiam se beneficiar mais com os jogos, por uma perspectiva motora, social, ou simplesmente pela prática de atividade física.

\section{Outro professor de Educação Física também mencionou que}

[...] os alunos que foram indicados para o projeto foram os que, por qualquer motivo, tentam não participar da aula de Educação Física. [...] Algumas crianças não gostam de correr, é algo que não faz parte de sua vida diária e não lhes dá prazer. [O laboratório de exergames] foi uma forma de motiválos em uma atividade que é diferente da prática mais comum de jogos coletivos da Educação Física.

Durante um período de três meses, dois pesquisadores estiveram envolvidos na coleta de dados. Um deles foi o instrutor que promoveu as atividades e aulas com seis grupos de alunos diferentes, entre as idades de oito e 14 anos. $O$ segundo pesquisador tomou notas sobre a interação dos alunos e as práticas realizadas durante as aulas. As aulas regulares 
de Educação Física aconteceram duas vezes por semana, de modo que os estudantes que participaram do projeto foram dispensados de uma dessas aulas a fim de poderem realizar uma prática diferente com os exergames. Portanto, os alunos tiveram uma aula regular de Educação Física e a segunda aula no laboratório de exergames, todas as semanas durante os três meses do projeto.

A estrutura das aulas foi organizada para oferecer atividades para quatro alunos de cada vez, usando os jogos Kinect Sports e Kinect Adventures. Na maioria das práticas iniciais, os alunos foram convidados a utilizar determinados jogos escolhidos pelo instrutor, com o objetivo de familiarizá-los com todos os jogos disponíveis, e também para variar as práticas em termos de nível de intensidade física. Após o primeiro mês, algumas vezes os próprios alunos tiveram a possibilidade de escolher os jogos que desejavam utilizar, dentre os jogos já elencados para o projeto.

O observador não participante (segundo pesquisador) não se envolveu diretamente nas atividades realizadas com os alunos. Seu papel foi apenas de registrar as interações entre os estudantes, anotando diálogos e relatando situações que demonstravam envolvimento e colaboração entre os participantes. Os registros foram feitos no laboratório e mais tarde ampliados em relatórios escritos sobre o que se pôde ouvir, ver e presenciar durante 0 momento de observação, de acordo com protocolos definidos por Bogdan e Biklen (2006). Já o pesquisador que trabalhou em abordagem participante interagiu com os alunos, explicando os jogos e dando-lhes dicas sobre como usá-los. Foi possível observar a dinâmica e os diferentes tipos de interação social entre os participantes.

Comportamentos e ações relevantes observados durante os encontros com os alunos foram descritos nas duas subseções a seguir, considerando os aspectos de engajamento na prática de atividade física e interação social/colaboração. Os professores de Educação Física desses estudantes também foram entrevistados para que se pudesse conhecer a sua percepção sobre o projeto e como os alunos perceberam as atividades no laboratório.

\subsection{Engajamento na prática de atividade física}

Em um contexto educacional, Chapman (2003) define o engajamento como a intensidade e a qualidade emocional com que os alunos se envolvem em atividades de aprendizagem. Os alunos que estão engajados nas atividades colocam esforço e concentração na realização das tarefas, demostrando envolvimento comportamental e entusiasmo. Natriello (1984) destaca que os aspectos relacionados ao engajamento fazem com que os estudantes fiquem mais dispostos a se envolver com atividades de rotina. A partir dessas considerações, durante o projeto, os instrutores tentaram identificar evidências de engajamento nas práticas com os exergames focando em três aspectos específicos, cada um deles detalhado a seguir.

\subsubsection{Disposição}

O aspecto disposição está relacionado à atitude espontânea do estudante em se envolver nas atividades propostas. Algumas das falas dos estudantes demonstraram claramente esse aspecto, como o comentário feito pelo aluno $\mathrm{A}$, que mostra como sua disposição em participar das aulas da Educação Física mudou com relação às práticas no laboratório de exergames: 
[...] Eu não gosto da Educação Física e daí era uma maneira que eu ia para a aula. Curti muito a prática. Porque bem ou mal tu acaba se exercitando mesmo e fazendo as atividades que tem pra fazer, só que de uma forma bem mais divertida, bem mais virtual.

O comentário feito pelo aluno B também evidencia sua visão positiva das práticas desenvolvidas no laboratório e sua disposição em participar das atividades:

[...] Eu gostei bastante. Não é aquela coisa chata. É que eu não gosto de jogar futebol, fazer os esportes ali fora, mas eu curto bastante jogar videogame. Aí achei uma ideia bem bolada, misturar o exercício físico com o videogame, porque aí une o útil ao agradável.

As evidências coletadas a partir das entrevistas com os professores de Educação Física da escola também permitiram observar que a introdução dos exergames foi positiva quanto à disposição dos alunos em participar das aulas:

[...] foi possível ver um efeito positivo em alguns dos alunos que foram selecionados para participar da pesquisa. Começamos a perceber uma motivação mais forte [desses alunos] para participar das aulas regulares de Educação Física. Ele pode ter ajudado estes alunos para conhecer seus próprios corpos.

Rikard e Banville (2006) colocam que é frequente os alunos terem uma boa disposição para a prática da Educação Física, sendo esta ligada principalmente ao fator diversão. Contudo, alguns estudos mostram que muitos estudantes não gostam das práticas propostas ou consideram a Educação Física entediante em razão de atividades repetitivas (KROUSCAS, 1999). Assim, a criação de novos espaços que possibilitem o desenvolvimento de novas práticas, como o laboratório de exergames, torna-se um fator importante.

\subsubsection{Entusiasmo}

Enquanto o conceito de disposição está relacionado à espontaneidade dos estudantes em se envolver em determinada atividade, o conceito de entusiasmo vai um pouco além, buscando demonstrar o grau de motivação e envolvimento dos alunos. Muitas das falas dos estudantes demonstraram seu entusiasmo com as atividades. A aluna C comentou: "[...] esse jogo de futebol é muito legal porque eu posso jogá-lo" (fazendo uma alusão à falta de possibilidade que meninas têm para jogar este esporte em particular). Já 0 aluno $D$ falou efusivamente ao jogar boliche: "[...] Strike! Mais um! Estou realmente bom neste jogo". Um pouco mais tarde, o aluno pergunta se ele e seus colegas de classe poderiam jogar novamente.

Interjeições como essas, seguidas de sorrisos e danças comemorativas, eram frequentes no laboratório, demonstrando a percepção positiva dos alunos com o desenvolvimento das atividades com os exergames. Ficou claro desde o início do projeto que os alunos se sentiam entusiasmados com o uso dos jogos, o que está alinhado ao fato de que esses eram alunos que começaram suas primeiras descobertas do mundo já interagindo com tecnologias (SUN, 2013).

$\mathrm{Na}$ entrevista com os professores, um deles trouxe a questão motivacional quando questionado sobre o uso da tecnologia na Educação Física. De acordo com as percepções do educador, em razão da demanda que os estudantes têm no uso da tecnologia no dia a dia, ele considera importante o emprego de novos artefatos pela Educação Física, principalmente 
por questões motivacionais. Manley e Whitaker (2011) também reforçam essas ideias ao apontar que o uso de exergames pode ser considerado uma novidade, e abordagens de ensino inovadoras podem aumentar o entusiasmo dos estudantes pelo conteúdo da disciplina.

\subsubsection{Interesse situacional}

Guthrie e Wigfield (1997) definem o interesse como um aspecto com facetas distintas, entre elas a de interesse pessoal e a de interesse situacional. Enquanto o interesse pessoal se refere aos sentimentos mais estáveis e duradouros, o interesse situacional é mais espontâneo e transitório, estando relacionado ao desenvolvimento de determinadas tarefas em contextos específicos. A observação aqui realizada teve como foco o interesse situacional, sendo este 0 tipo de interesse que pode ser influenciado pelo professor ou por propostas de atividades

Alguns comentários dos estudantes levaram a crer que o emprego dos jogos no laboratório de exergames despertou o interesse situacional dos estudantes, como apontado pelo aluno F: "[...] Era um jeito diferente de praticar atividade física. Achei bem interessante, uma coisa nova. Poucas pessoas têm isso em casa, então achei bem interessante, bem atrativo". Outro comentário reforça esse aspecto: "[...] Eu achei as atividades bem interessantes porque uniam o videogame com um modo diferente de prática do esporte em si, formando uma coisa lúdica".

O aluno G também argumentou: "[...] Um dos jogos eu achei bem útil, que mostrava como fazer os exercícios, como o abdominal e as musculaturas que trabalhavam nesses exercícios. Achei bem interessante, tu aprendia junto".

Os dados coletados durante os três meses demonstraram que os estudantes que aderiram ao projeto se mostraram interessados nas propostas de atividades no laboratório, demonstrando frequentemente curiosidade e trazendo questões sobre os jogos, as atividades, os esportes e exercícios que tinham que fazer.

Nas entrevistas com os professores de Educação Física, muitas das falas evidenciaram 0 interesse situacional dos estudantes:

[...] Os alunos pensaram, a princípio, que eles iriam fugir de aulas de Educação Física para jogar videogames, [...], mas no decorrer do projeto, pudemos sentir motivação. [...] Se você considerar um menino que não teve uma vida muito ativa, um estudante que passa muito tempo dentro de casa, ele começou a sentir os movimentos do corpo, mover-se, jogar, dar um soco (referindo-se ao boxe no jogo Kinect Sports). [...] talvez ele tenha começado a descobrir o seu próprio corpo.

O mesmo professor ainda comentou sobre o uso dos exergames e de tecnologias nas aulas: "[...] Houve uma motivação inicial por parte dos alunos, [...] podemos falar em um primeiro momento do próprio XBOX, ou a gente pode falar de um tablet para demonstrar a parte didática de um jogo".

Tais comentários evidenciam que a introdução das atividades empregando tecnologia no laboratório de exergames alterou o interesse situacional dos estudantes, trazendo implicações positivas mesmo àqueles que normalmente não gostam de aderir às práticas propostas nas aulas regulares de Educação Física. Resultados apresentados por Baracho, Gripp e Lima (2012) também apontam que, no cenário da cultura digital contemporânea, existe uma necessidade premente de ampliação das possibilidades das práticas corporais, sendo papel da Educação Física explorar essas possibilidades. 
A implementação do laboratório de exergames também vai ao encontro de outros estudos que indicam que um número grande de estudantes apreciariam ter mais variedade e novidade nas propostas de atividades da Educação Física (RIKARD; BANVILLE, 2006).

\subsection{Interação social e colaboração}

O processo de colaboração é diretamente ligado à comunicação, à confiança e ao desenvolvimento de habilidades de interação social positivas (ORLICK, 1977). É por meio da colaboração que os estudantes aprendem a compartilhar, aprendem a se colocar no lugar do outro e assim desenvolvem habilidades de trabalho em grupo.

Através da observação de como os alunos interagiram uns com os outros, registramos como a colaboração e a resolução de conflitos aconteceram entre os participantes. Foi perceptível a vontade natural dos alunos de ajudar seus colegas que demonstravam mais dificuldade em jogar. $\mathrm{O}$ apoio era dado geralmente com instruções verbais e regras lógicas do jogo. Além disso, a atitude dos alunos mais confiantes serviu de modelo para aqueles que precisavam de mais apoio para as atividades. Também foi perceptível que os alunos passaram a colaborar mais durante as aulas. Essa colaboração teve um impacto positivo em todos os alunos que tinham dificuldade nas práticas iniciais. Pouco a pouco, eles começaram a se sentir mais integrados no grupo e confiantes em realizar as atividades propostas. Foi possível observar que, em grupos pequenos, era mais fácil para os alunos entrarem em contato uns com os outros. Observações de incentivo feitas pelos participantes também foram consideradas como um outro tipo de evidência de interação social:

- Aluno I: buscando motivar outros colegas para conseguirem mais pontos jogando boliche: "[...] Vamos lá, tu consegues!"; "[...] Tu vais ter mais pontos, te esforça!".

- Aluno J: torcendo por outros colegas enquanto aguardava sua vez de jogar: "[...] Vai em frente, tu estás bem!".

Esse tipo de fala foi muito frequente durante as aulas no laboratório de exergames. Outra evidência de que as práticas no laboratório promoveram a interação social é que, paulatinamente, os estudantes começaram a auto-organizar seus grupos para jogar. Eles começaram a definir os pares, a ordem dos jogadores e os jogos preferidos. Eles também começaram a resolver pequenos desentendimentos por conta própria, sem qualquer intervenção do instrutor. Houve até um episódio em que um aluno disse para o outro que as atividades não deveriam ser tão competitivas, a fim de "acalmar os ânimos" e manter a cordialidade entre todos. A resolução de conflitos é tomada como responsabilidade do educador, e envolve diferentes estratégias para contornar tais situações (STIHEC; VIDEMSEK; VRBNJAK, 2011). Uma dessas é a colaboração e escuta do outro, estratégia utilizada pelo estudante na tentativa de resolver 0 desentendimento que surgia entre o grupo.

A colaboração entre alunos de sexo oposto também pôde ser observada: os alunos $\mathrm{H}, \mathrm{I}$, J e K se organizaram para fazer pares mistos para jogar alguns dos jogos, apontando que, nas aulas de Educação Física regular, as duplas sempre são entre dois meninos ou entre duas meninas.

Alguns dos estudantes também mostraram progresso especialmente em relação à timidez e à introspecção. Como os grupos eram menores (máximo de quatro alunos), foi 
possível observar que os alunos se sentiam mais integrados do que em grupos maiores, mais frequentes nas aulas de Educação Física regular.

Nas entrevistas gravadas com os professores de Educação Física, alguns dos comentários mostraram como o projeto ajudou os alunos em suas práticas colaborativas e sociais. Um dos professores de Educação Física disse:

[...] Em relação à interação social, é certo que em aulas de Educação Física os alunos começam a interagir mais, mas eles estão fazendo isso de qualquer maneira [nessa idade]. Nós começamos a observar uma atitude mais extrovertida [dos alunos participantes do projeto], menos relutantes em se expor. As pessoas se comunicam através de seus corpos. No momento de eu passar a bola para alguém sem qualquer medo de cometer um erro, ou eu pedir uma bola só de olhar para o outro, eu estou me comunicando.

O mesmo professor observou, porém, que a timidez é um aspecto difícil de lidar. Ele ressaltou que ele não teria evidências suficientes para fazer uma declaração definitiva sobre mudanças permanentes a respeito da introversão dos alunos. Mesmo assim, é importante destacar um comentário do instrutor do laboratório de exergames em sua entrevista:

[...] Foi evidente que os alunos introvertidos se tornaram mais desenvoltos e desinibidos na medida em que o projeto avançou, e em algum ponto passaram a dar dicas sobre os jogos aos demais. Os alunos que não eram muito ativos nas aulas de Educação Física se mostraram muito motivados no laboratório, também demonstrando mais interesse por temas ligados ao esporte e saúde.

Outro comentário relevante do professor demonstrou o potencial do laboratório em engajar os estudantes, mesmo os que têm dificuldade em participar das práticas regulares da Educação Física:

[...] Nós estávamos comentando outro dia sobre um estudante que estava iniciando em nossa escola, muito tímido, sempre só. Nós o convidamos a participar do projeto, e sua melhora foi visível. É difícil dizer com certeza se o laboratório exergames foi o responsável pela mudança, mas seria muita coincidência. [...] 0 projeto não está mais em curso, mas o aluno começou 0 ano muito bem. Ele tem limitações motoras, técnicas, mas hoje ele tenta participar [das aulas] do seu próprio jeito. Eu acredito que estes resultados estão relacionados com o trabalho que tem sido feito no laboratório de exergames.

O desenvolvimento de habilidades sociais nos estudantes é tópico de estudo de muitos pesquisadores na Educação Física. Resultados de algumas destas pesquisas mostram que atividades específicas nas aulas de Educação Física podem melhorar as habilidades sociais dos estudantes e levá-los à preferir atividades em grupo (GOUDAS; MAGOTSIOU, 2009). Entende-se, a partir dos dados da pesquisa aqui apresentada, que as atividades desenvolvidas no laboratório de exergames também facilitaram a interação social entre os alunos, ajudandoos a melhorar suas habilidades sociais.

\section{CONSIDERAÇÕES FINAIS}

A principal contribuição deste projeto foi mostrar como um laboratório de exergames pode ser uma ferramenta de apoio às aulas regulares Educação Física, 
oferecendo uma alternativa para alunos desmotivados nas aulas da disciplina. Foram apresentadas evidências na pesquisa de que as práticas semanais no laboratório de exergames complementaram as aulas de Educação Física, envolvendo esses alunos na prática de atividade física e trabalhando também suas habilidades sociais. Em relação à prática regular de atividades físicas, os resultados mostraram que os exergames podem proporcionar situações em que os alunos podem aumentar a regularidade das suas práticas, envolvendo-os nas atividades propostas em aula. Esses resultados estão alinhados a resultados de outros trabalhos que mostram que, por meio do envolvimento sistemático de pessoas em atividades de recreação e esporte com extensão e intensidade adequadas, efeitos significativos podem ser alcançados (MAVRIC et al., 2014). Outros estudos também têm demonstrado que a introdução de exergames nas aulas de Educação Física pode influenciar as atitudes e intenções dos alunos, assim como a prática sistemática de exercícios físicos (LWIN; MALIK, 2012).

Os resultados da pesquisa realizada também permitiram concluir que a colaboração aconteceu no laboratório de exergames de muitas maneiras diferentes, com os alunos apoiando uns aos outros ao longo de suas práticas e se tornando mais confiantes com seu desempenho no grupo. Depois de se envolverem no projeto, movidos pela novidade da prática de atividade física com os jogos, os alunos também demonstraram interesse em aprender mais sobre diferentes esportes e sobre a importância da prática de atividade física regular.

Tais resultados apontam para um quadro positivo quanto à introdução dos exergames em práticas escolares, acompanhando a tendência global de emprego de novas tecnologias na educação. Contudo, Vaghetti e Botelho (2010) destacam que, para que os exergames possam ser introduzidos em práticas regulares em aulas de Educação Física, seria necessário que os professores pudessem conhecê-los de maneira mais sistemática e aprofundada. 0 emprego desses artefatos em atividades de aprendizagem deve ser planejado para que possam atender às expectativas do currículo, levando em consideração especificidades dos estudantes.

Como sugestão para trabalhos futuros, temos como objetivo investigar como os exergames podem ajudar alunos a monitorar e entender melhor sua própria condição física por meio do acompanhamento de seu desempenho nos jogos.

\section{REFERÊNCIAS}

BARACHO, Ana Flávia de Oliveira; GRIPP, Fernando Joaquim; LIMA, Márcio Roberto de. Os exergames e a educação física escolar na cultura digital. Revista Brasileira de Ciências do Esporte, Florianópolis, v. 34, n. 1, p. 111-126, jan./mar. 2012.

BARANOWSKI, Tom et al. Playing for real: video games and stories for health-related behavior change. American Journal of Preventive Medicine, Philadelphia, v. 1, n. 34, p. 74-82, jan. 2008.

BOGDAN, Robert; BIKLEN, Sari Knopp. Qualitative investigation in education: an introduction for the theory and methods. New York: Pearson, 2006.

CARLSON, Teresa. We hate gym: student alienation from physical education. Journal of 
CHAPMAN, Elaine. Alternative approaches to assessing student engagement rates. Practical Assessment, Research \& Evaluation Journal, New York, v. 13, n. 8, p. 251-263, 2003.

CHIN, Mai Paw et al. The motivation of children to play an active video game. Journal of Science and Medicine in Sport, New York, v. 2, n. 11, p. 163-166, 2008.

DISHMAN, Rodney et al. Enjoyment mediates effects of a school-based physical-activity intervention. Medicine and Science in Sports and Exercise, Lippincott, v. 3, n. 37, p. 478-487, 2005.

ENNIS, Catherine. Students' experiences in sport-based physical education: more than apologies are necessary. Quest, London, v. 1, n. 48, p. 453-456, 1996.

FERY, Yves-Andre; PONSERRE, Sylvain. Enhancing the control of force in putting by video game training. Journal of Ergonomics, London, v. 12, n. 44, p. 1025-1037, 2011.

FOGEL, Victoria et al. The effects of exergaming on physical activity among inactive children in a physical education classroom. Journal of Applied Behavior Analysis, Melbourne, v. 4, n. 43, p. 591-600, 2010.

GOUDAS, Marios; MAGOTSIOU, Evmorfia. The effects of a cooperative physical education program on students' social skills. Journal of Applied Sport Psychology, London, v. 21, n. 3 , p. 356-364, 2009.

GREGORIADIS, Athanasios; GRAMMATIKOPOULOS, Vasilis; ZACHOPOULOU, Evridiki. Evaluating preschoolers' social skills: the impact of a physical education program from the parents' perspective. International Journal of Humanities and Social Science, Los Angeles, v. 10, n. 3, p. 40-51, 2004.

GUTHRIE, John; WIGFIELD, Allan. Reading engagement: a rationale for theory and teaching. In: GUTHRIE, John; WIGFIELD, Allan. Reading Engagement: Motivating Readers Through Integrated Instruction. Newark: International Reading Association, 1997, p. 14-43.

HAYES, Elisabeth; SILBERMAN, Lauren. Incorporating video games into physical education. Journal of Physical Education Recreation and Dance, London, v. 3, n. 78, p. 18-24, 2007.

KROUSCAS, James Athan Jr. Middle school students' attitudes toward a physical education program. 1999. Tese (Doutorado) - Programa de Pós-Graduação do Departamento de Ensino e Aprendizagem, do Instituto Politécnico e Universidade Estadual de Virgínia, Blacksburg, 1999.

LIEBERMAN, Debra. What can we learn from playing interactive games? In: VORDERER, Peter; BRYANT, Jennings. Playing video games: motives, responses, and consequences. London: Routledge, 2006. p. 447-470.

LWIN, May; MALIK, Shelly. The efficacy of exergames-incorporated physical education lessons in influencing drivers of physical activity: a comparison of children and pre-adolescents.

Psychology of Sport and Exercise, New York, v. 1, n. 13, p. 756-760, 2012.

MANLEY, Andrew; WHITAKER, Lisa. Wii-learning: using active video games to enhance the learning experience of undergraduate sport psychology students. Sport \& Exercise Psychology Review, London, v. 7, n. 2, p. 45-55, 2011.

MAVRIC, Fahrudin et al. The effects of regular physical exercise on the human body. Physical Culture, Belgrade, v. 1, n. 68, p. 29-38, 2014. 
MUELLER, Florian; AGAMANOLIS, Stefan; PICARD, Rosalind. Exertion interfaces: Sports over a Distance for Social Bonding and Fun. In: CONFERÊNCIA HUMAN FACTORS IN COMPUTING SYSTEMS, 20., 2003, New York. Anais... New York: ACM Press, 2003. p. 561-568.

NATRIELLO, Gary. Problems in the evaluation of students and student disengagement from secondary schools. Journal of Research and Development in Education, Athens, v. 17, n. 4, p. 14-24, 1984.

ORLICK, Terry. The cooperative sports and games book: challenge without competition. New York: Pantheon, 1978.

PENG, Wei; LIN, Jih-Hsuan; CROUSE, Julia. Is playing exergames really exercising? A Metaanalysis of energy expenditure in active video games. Cyberpsychology, Behavior and Social Networking, New Rochelle, v. 14, n. 11, p. 681-688, 2011.

RIKARD, Linda; BANVILLE, Dominique. High school student attitudes about physical education. Sport, Education and Society, London, v. 11, n. 4, p. 385-400, 2006.

SHAYNE Rachel et al. The effects of exergaming on physical activity in a third-grade physical education class. Journal of Applied Behavior Analysis, London, v. 1, n. 45, p. 211-215, 2012.

STAIANO, Amanda; CALVERT, Sandra. Exergames for physical education courses: physical, social and cognitive benefits. Child Development Perspective Journal, London, v. 2, n. 5, p. 93-98, 2011.

STELZER, Johannes et al. Attitudes toward physical education: a study of high school students from four countries: Austria, Czech Republic, England and USA. College Student Journal, Birmingham, v. 38, n. 2, p. 171-178, 2004.

STIHEC, Joze; VIDEMSEC, Mateja; VRBNJAK, Sasa. Analysis of conflict resolution in physical education depending on a teacher's gender. Acta Gymnica, Olomouc, v. 41, n. 2, p. 35-43, 2011.

STRONG, William et al. Evidence based physical activity for school-aged youth. The Journal of Pediatrics, Cincinnati, v. 6, n. 146, p. 732-737, 2005.

SUN, Haichun. Impact of Exergames on physical activity and motivation in elementary school students: a follow-up study. Journal of Sport and Health Science, London, v. 3, n. 2, p. 138145, 2013.

TROUT, Josh; CHRISTIE, Brett, J. Interactive video games in physical education: rather than contribute to a sedentary lifestyle, these games demand activity from the players. Journal of Physical Education, Recreation and Dance, London, v. 5, n. 78, p. 29-34, 2007.

TRUDEAU, François; SHEPHARD, Roy. Physical education, school physical activity, school sports and academic performance. International Journal of Behavioral Nutrition and Physical Activity, London, v. 1, n. 1, p. 5-10, 2008.

US DEPARTMENT OF HEALTH AND HUMAN SERVICES. Youth physical activity: the role of schools. Atlanta, GA, Agosto, 2009. Disponível em: <www.cdc.gov/healthyyouth/physicalactivity/ toolkit/factsheet_pa_guidelines_schools.pdf>. Acesso em: 10 out. 2014.

VAGHETTI, César Augusto Otero; BOTELHO, Silvia Silva da Costa. Ambientes virtuais de aprendizagem na educação física: uma revisão sobre a utilização de exergames. Ciências \& Cognição, Rio de Janeiro, v. 15, n. 1, p. 76-88, 2010. 
WIEMEYER, Josef. Serious games - the challenges for computer science in sport. International Journal of Computer Science in Sport, Viena, v. 9, n. 2, p. 65-75, 2010.

WILLIAMS, David et al. Perceived enjoyment moderates the efficacy of an individually tailored physical activity intervention. Journal of Sport Exercise and Psychology, Champaign, v. 3, n. 28, p. 300-309, 2006.

Apoio: FAPERGS/CAPES (Processo No. 0420-2551/14-0) e CNPq (Processo No. 490223/2012-5). 